\title{
Detection of Nitrite by flow injection analysis using a novel Paired Emitter-Detector Diode (PEDD) as a photometric Detector
}

\author{
Martina O’ Toole, Roderick Shepherd, King-Tong Lau and Dermot Diamond* \\ Adaptive Sensors Group, National Centre for Sensor Research, School of Chemical \\ Sciences, Dublin City University, Dublin 9.
}

\begin{abstract}
An inexpensive flow injection analysis system for determining low concentration levels of nitrite employing the Griess reagent spectrophotometric method is reported. The novel photometric detector applied within this manifold is a highly sensitive, low cost, miniaturized light emitting diode (LED) based flow detector. This colorimetric detector employs two LEDs, operating one as a light source and the other as a light detector. The emitter LED is forward biased and the detector reverse biased. The emitter and detector LED had a $\lambda_{\max }$ of $530 \mathrm{~nm}$ and $623 \mathrm{~nm}$ respectively. The emission spectrum of the emitter LED efficiently overlapped with the absorbance spectrum of $9 \mu \mathrm{M} \mathrm{NO}_{2}$ and Griess reagent complex. A simple timer circuit measures the time taken for the photocurrent generated by the emitter LED to discharge the detector LED from $5 \mathrm{~V}$ (logic 1) to $1.7 \mathrm{~V}$ (logic 0).

The Griess reagent method employed for nitrite determination is based on the formation of an azo dye, the intensity of which is directly related to nitrite concentration. The linear range, reproducibility and limit of detection were investigated. Detection limits in the nanomolar range were achieved using the Paired Emitter-Detector Diode (PEDD) flow analysis device. For a comparative study the linear range and limit of detection were also investigated using a platewell reader. Higher sensitivity and improved precision were obtained from the PEDD compared to the commercially available plate well reader.
\end{abstract}

\section{KEYWORDS}

Light emitting diodes, Optical sensing, Nitrite Detection, Griess Reaction and Flow Analysis

\section{INTRODUCTION}

Nitrite is widely found in soil, natural water and food and produces nitrosamines in the human body through its reaction with amines or amides, which are carcinogenic ${ }^{1}$. An additional human threat posed by the presence of nitrite at high concentration in the blood is its reaction with iron (III) of the haemoglobin, forming methemoglobin, which has no oxygen carrying ability ${ }^{2}$. Many analytical methods have been proposed for the detection of nitrite ${ }^{2-10}$. Most of the methods employed for the determination of nitrite are based on the colorimetric determination of nitrite with diazocoupling reaction. In 1963 Sawicki et al. published a review of over 50 spectrophotometric methods for the determination of nitrite ${ }^{11}$. In 1879 , Griess proposed the reaction of sulfanilic acid with 1-naphthylamine in the presence of nitrites and sulfuric acid and proposed that it could be used for the determination of nitrites ${ }^{12}$. Modifications of the original Griess method have been investigated and it is still one of the most commonly employed colorimetric method for the detection of nitrites ${ }^{13-20}$.

The reaction mechanism for the detection of nitrite employing the Griess reaction method is based on the conversion of sulfanilic acid (Reagent A) to a diazonium salt by reaction with nitrite in acid solution. The diazonium salt is then

\footnotetext{
* Correspondence author: Tel.: +353-1-7005404; fax: +353-1-7008002 Email: dermot.diamond@ dcu.ie
} 
coupled to $N$-(1-naphthyl)ethylenediamine (NED) (Reagent B), forming an azo dye that can be spectrophotometrically quantified based on its absorbance at $547 \mathrm{~nm}$ (Figure 1).

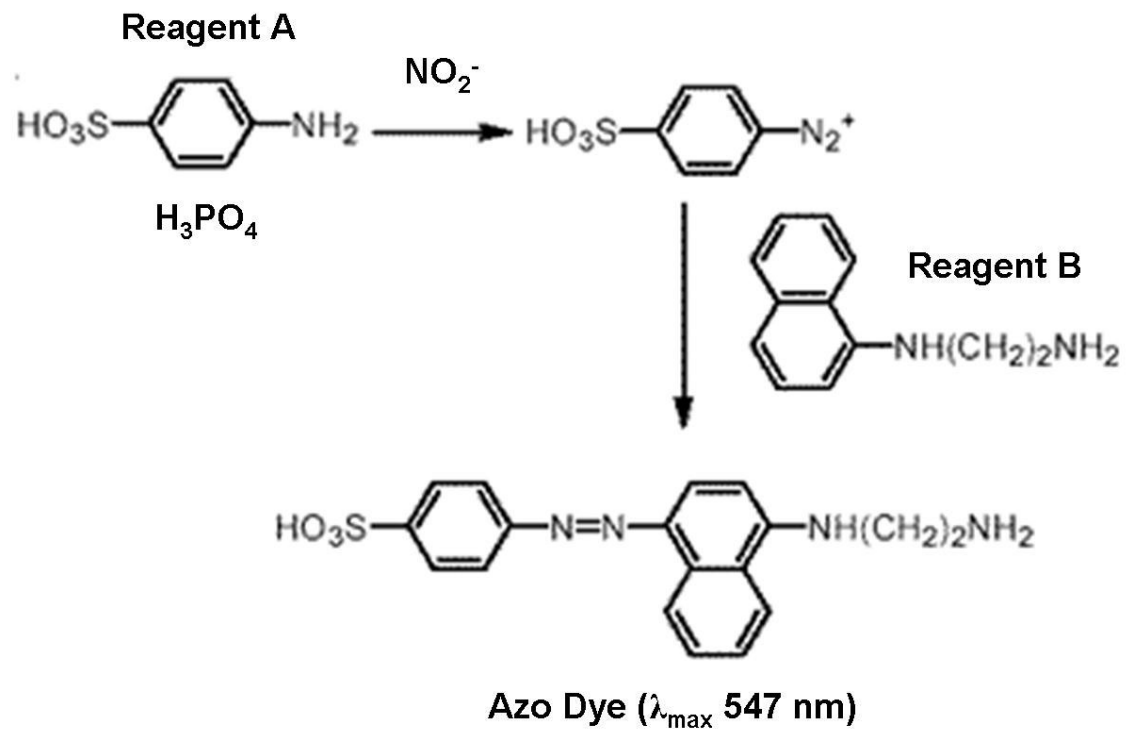

Figure 1 Mechanism of nitrite detection employing the Griess reaction method.

Miniature, portable, robust, accurate and inexpensive environmental monitoring devices are required for autonomous field deployment monitoring ${ }^{21}$. In most microanalytical systems neither the light source nor the photodetector is integrated into the same substrate as the fluidic channel network. This is because the integration of all components necessary for performing a total chemical analysis is very complex ${ }^{22}$. Several optical devices have been proposed for the detection of nitrites, however often the components of the device are external (i.e. not fully integrated into the

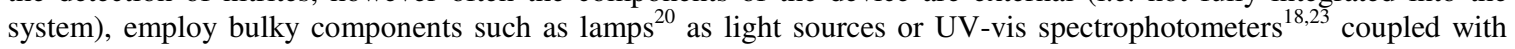
fiber optic cables as the detector.

In this paper we present the detection of nitrite using a novel detector, which has the light source, detector and fluidic channel fully integrated. Optimum conditions such as time allowed for colour formation, reagent to sample ratio and flow rate were investigated for the spectrophotometric method. The reproducibility, linear range and limit of detection were determined. A comparative study carried out using a commercially available platewell reader was carried out. Under optimised conditions the low cost PEDD detector ( $\sim \$ 1)$ displayed higher sensitivity and improved precision compared to the commercially available platewell reader.

\section{EXPERIMENTAL}

\subsection{Chemicals and reagents}

All solutions were prepared from analytical grade chemicals. Deionised water obtained from a Millipore Milli-Q water purification system was used for all analysis. New stock solutions were prepared weekly. Reagent A and B were stored refrigerated at $2-6^{0} \mathrm{C}$, protected from light.

Phosphoric acid $\left(\mathrm{H}_{3} \mathrm{PO}_{4}, \mathrm{BDH}^{\mathrm{TM}}\right.$, Poole, UK)

A $5 \%$ phosphoric acid solution was prepared by dissolving $5 \mathrm{~g}$ of $\mathrm{H}_{3} \mathrm{PO}_{4}$ in $100 \mathrm{~mL}$ of deionised water

Reagent A: Sulfanilic acid $\left(\mathrm{C}_{6} \mathrm{H}_{7} \mathrm{NO}_{3} \mathrm{~S}\right.$, Sigma-Aldrich, Dublin, Ireland)

A $1 \%$ sulfanilic acid solution was prepared by dissolving $1 \mathrm{~g}$ of sulfanilic acid in $100 \mathrm{~mL}$ of a $5 \%$ phosphoric acid solution. 
Reagent B: N-(1-naphthyl)ethylenediamine dihydrochloride (NED), $\left(\mathrm{C}_{12} \mathrm{H}_{14} \mathrm{~N}_{2}\right.$. 2HCl, Sigma-Aldrich, Dublin, Ireland)

A $0.1 \%$ NED solution was prepared by dissolving $100 \mathrm{mg}$ of NED in $100 \mathrm{~mL}$ of deionised water.

Nitrite standard solution ( $\mathrm{NaNO}_{2}$, Sigma-Aldrich, Dublin, Ireland)

A $0.1 \mathrm{M}$ stock solution of sodium nitrite was prepared by dissolving $1.725 \mathrm{~g}$ of $\mathrm{NaNO}_{2}$ in $250 \mathrm{~mL}$ of deionised water.

\subsection{Measurement procedure}

\section{$\mu Q u a n t^{\mathrm{TM}}$ platewell reader}

The Griess reagent was prepared by mixing equal volumes of reagent A (sulfanilic acid) and reagent $\mathrm{B}$ ( $\mathrm{N}-(1$ naphthyl)ethylenedi-amine). The mixture was prepared daily. The colorimetric reagent $(0.2 \mathrm{~mL})$ was added to the nitrite standards $(3 \mathrm{~mL})$ and the solution was left to stand for $10 \mathrm{~min}$ for colorimetric development. The absorbance of the solution was measured comparatively using the $\left(\lambda_{\max } 530 \mathrm{~nm}\right)$ PEDD flow cell and a $\mu$ Quant ${ }^{\mathrm{TM}}$ platewell reader (Bio-Tek Instruments, Inc., USA).

\section{$\left(\lambda_{\max } 530 \mathrm{~nm}\right)$ PEDD}

The measurement is based on the following theoretical model (Equation 1), which has been derived by Lau et al $l^{24}$.

$\log (t)=\varepsilon C l+\log \left(t_{0}\right)$

Equation 1

where $l$ is the optical path length through the solution $(\mathrm{cm}), \varepsilon$ the molar extinction coefficient, $C$ the concentration of the absorbing species $\left(\mathrm{mol} \mathrm{L}^{-1}\right), t_{0}$ a constant that represents discharge time in the absence of the coloured species in solution $(\mu \mathrm{s})$ and $t$ is the discharge time in the presence of the coloured species in solution $(\mu \mathrm{s})$.

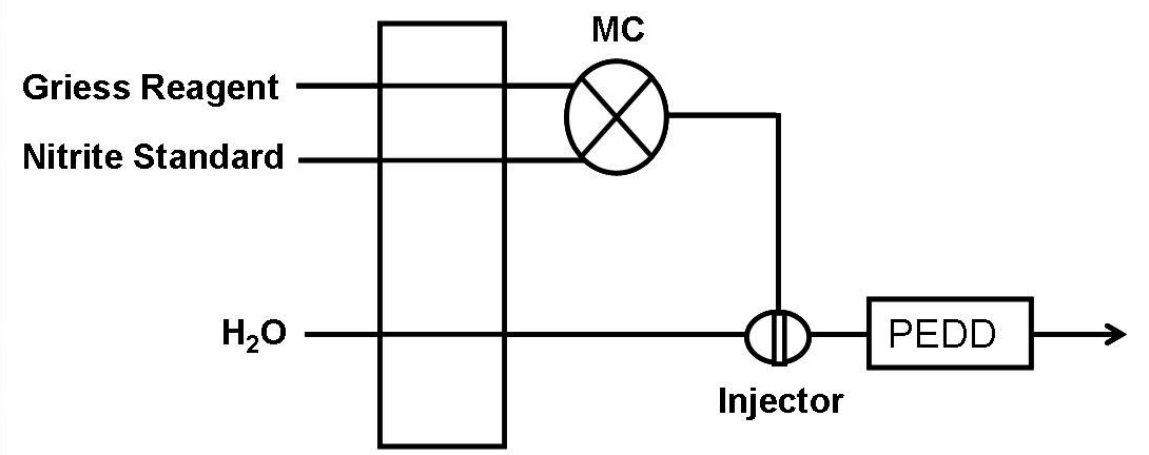

Figure 2 The flow analysis manifold employed for the determination of nitrite using a $\left(\lambda_{\max } 530 \mathrm{~nm}\right)$ PEDD. (MC $=$ mixing chamber).

The flow analysis manifold shown in Figure 2 consisted of a sample injector (Rheodyne, model 5020), and a Gilson (MiniPlus 3) peristaltic pump, (Anachem, UK). The Griess reagent and nitrite standard were pumped to the mixing chamber in reagent to sample ratio previously specified at a flow rate of $0.6 \mathrm{~mL} \mathrm{~min}^{-1}$. A $100 \mu \mathrm{L}$ injection sample loop was selected to inject the nitrite and Griess reagent complex into the carrier stream $\left(\mathrm{H}_{2} \mathrm{O}\right)$. The complex formed was then passed through the $\left(\lambda_{\max } 530 \mathrm{~nm}\right)$ PEDD flow cell.

\subsection{Fabrication of PEDD optical flow cell}

The integrated PEDD cell was fabricated as previously described ${ }^{25}$ using two $5 \mathrm{~mm}$ LEDs (Kingbright, Ireland) as shown in Figure 3. The detector used was a red LED $\left(\lambda_{\max }\right.$ at $\left.623 \mathrm{~nm}\right)$ which can detect any wavelength below this point. A green LED $\left(\lambda_{\max }\right.$ at $\left.530 \mathrm{~nm}\right)$ was used as the emitter LED. 


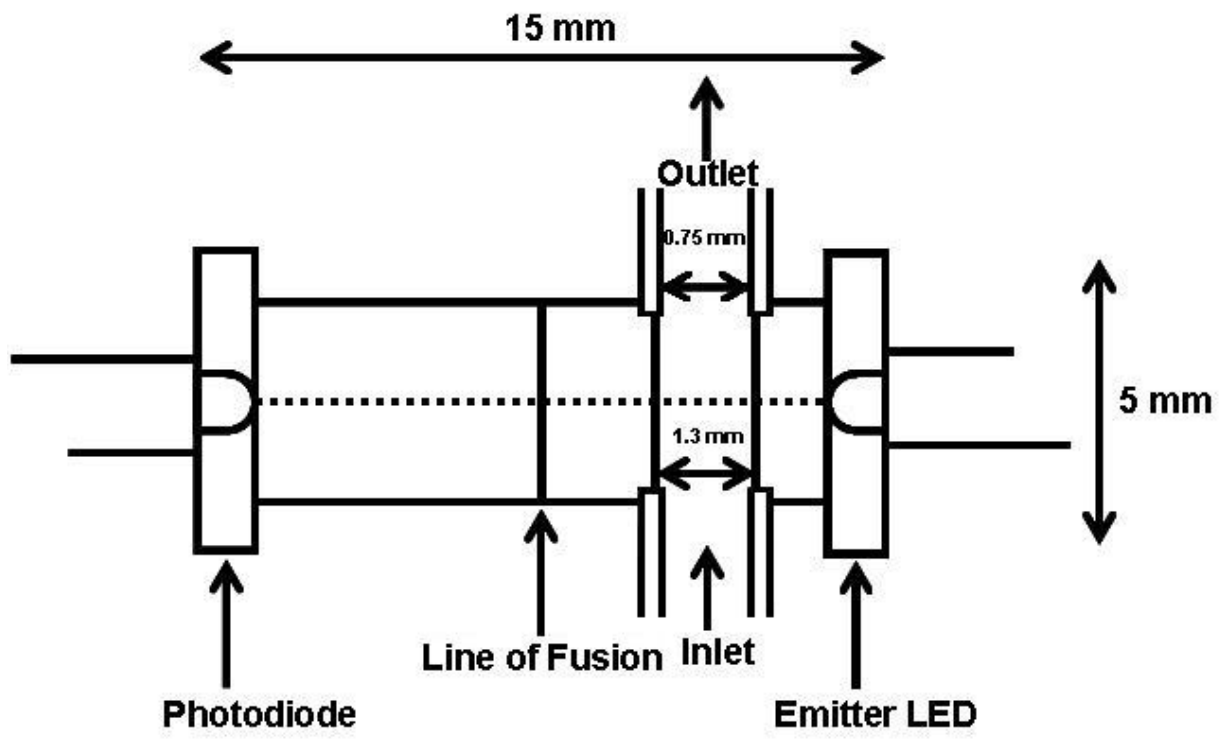

Figure 3 A schematic of the integrated PEDD flow analysis device used for colorimetric detection. The emitter LED had a $\lambda_{\max }$ of $530 \mathrm{~nm}$ and the detector LED had a $\lambda_{\max }$ of $623 \mathrm{~nm}$.

The light measurement technique and circuitry employed to drive the PEDD were as previously described ${ }^{25}$.

\section{RESULTS AND DISCUSSION}

\subsection{Optimization of flow system parameters}

\subsubsection{Wavelength}

The optimum wavelength to monitor the nitrite Griess reaction complex has been variously reported in the literature, typically citing the $\lambda_{\max }$ in the range ca. $526-563 \mathrm{~nm}^{15,17-20}$. The $\lambda_{\max }$ was therefore determined under our experimental conditions by obtaining the absorbance spectrum of $9 \mu \mathrm{M} \mathrm{NO} \mathrm{NO}_{2}$ using the $\mu$ Quant ${ }^{\mathrm{TM}}$ platewell reader (Bio-Tek Instruments, Inc., USA). The $\lambda_{\max }$ of the sample was found to be $547 \mathrm{~nm}$. An emitter LED with a $\lambda_{\max }$ of $530 \mathrm{~nm}$ was employed as the light source in the PEDD. An LED with a $\lambda_{\max }$ closer to $547 \mathrm{~nm}$ would provide increased sensitivity. The light intensity transmitted from the emitter LED $\left(\lambda_{\max } 530 \mathrm{~nm}\right)$ was measured with a detector LED that had a slightly smaller bandgap $\left(\lambda_{\max } 623 \mathrm{~nm}\right)$. The absorbance of the nitrite Griess reaction complex $\left(\lambda_{\max } 547 \mathrm{~nm}\right)$ as shown in Figure 4 efficiently overlaps with the emission spectrum of the emitter green LED and will therefore allow high sensitivity. The emission spectrum of the emitter LED was obtained by using Ocean Optics spectrometer (OOIBase $32^{\mathrm{TM}}$, Ocean Optics, Inc., Dunedin, USA). 


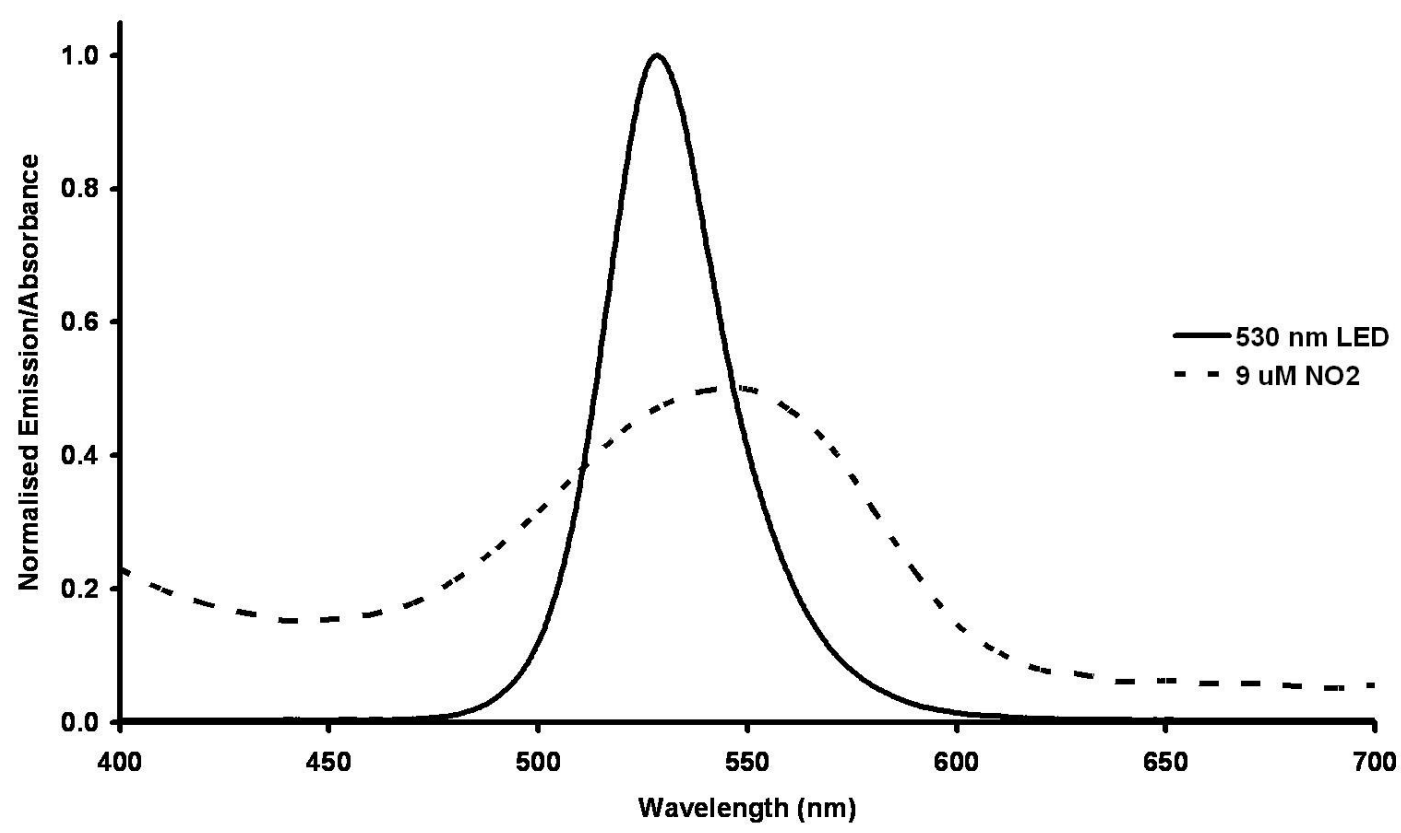

Figure 4 Emission spectrum $\left(\lambda_{\max } 530 \mathrm{~nm}\right)$ of the emitter LED (solid line) used in the integrated PEDD flow analysis device, the absorption spectrum $\left(\lambda_{\max } 623 \mathrm{~nm}\right)$ of $9 \mu \mathrm{M} \mathrm{NO}_{2}$ and Griess reagent (dashed line).

\subsubsection{Reagent to sample ratio}

The optimum reagent to sample ratio was determined by preparing a range of samples at a concentration of $2 \mu \mathrm{M} \mathrm{NO} \mathrm{N}_{2}$ with varying volumes of Griess reagent $(0.1-3 \mathrm{~mL})$ added. The normalised maximum absorbance $\left(\mathrm{R} / \mathrm{R}_{\max }\right)$ of each sample was recorded after 10 minutes using the $\mu$ Quant ${ }^{\mathrm{TM}}$ platewell reader and plotted against the volume of reagent added to $3 \mathrm{~mL}$ of sample. The reagent volume that provided the highest $\mathrm{R} / \mathrm{R}_{\max }$ i.e. the most intense colour was determined as $0.2: 3 \mathrm{v} / \mathrm{v}$ reagent to sample ratio (i.e. $0.2 \mathrm{~mL}$ reagent to $3 \mathrm{~mL}$ of sample). This was the reagent to sample ratio adopted throughout all remaining experiments.

\subsubsection{Kinetics study}

The development of the nitrite Griess reagent complex colour intensity was monitored for the detection of $2 \mu \mathrm{M} \mathrm{NO}$ using the $\mu$ Quant ${ }^{\mathrm{TM}}$ platewell reader by taking an absorbance measurement at a $\lambda_{\max }$ of $547 \mathrm{~nm}$ every 2 minutes for 40 minutes. The experiment was carried out in triplicate.

As shown in Figure 5 the colour formation increased rapidly until approximately 10 min after which the rate of increase ceased. This was the time allowed for colour development adopted for studies employing the $\mu$ Quant ${ }^{\mathrm{TM}}$ platewell reader 


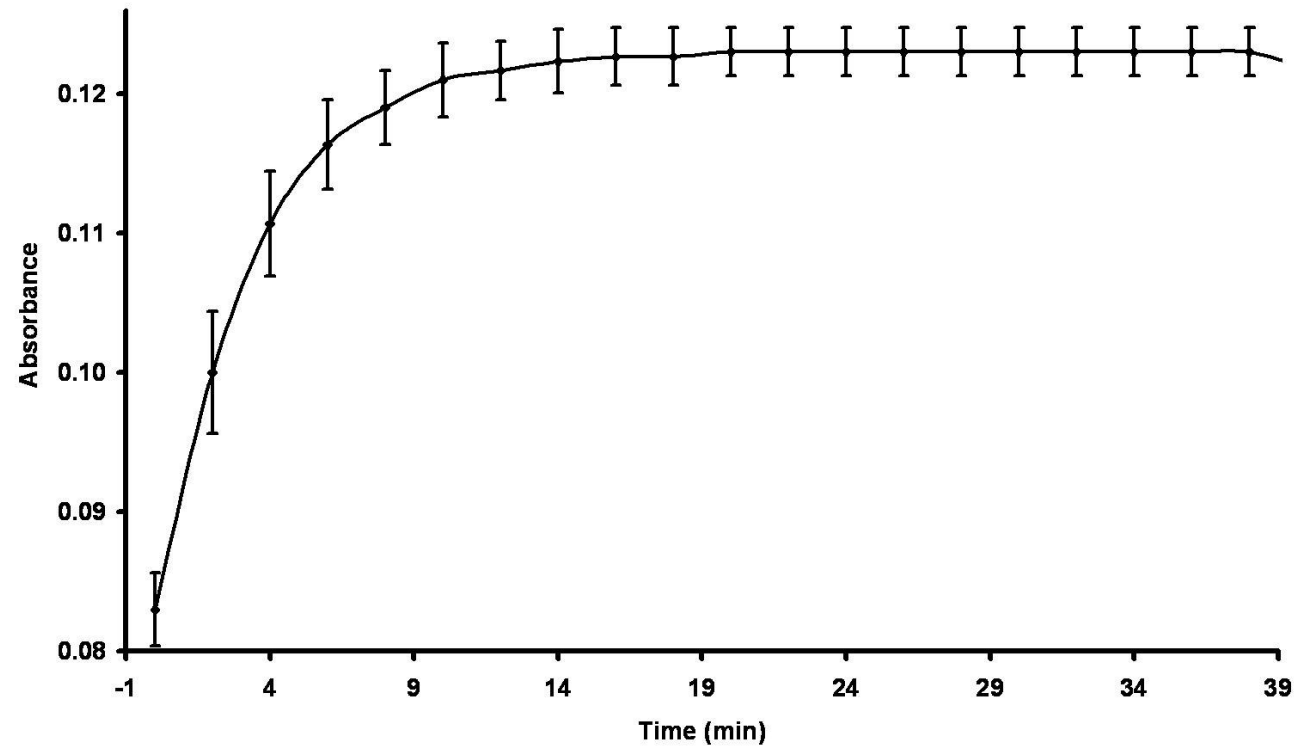

Figure 5 Kinetic study of the colour formation monitored at a wavelength $\lambda_{\max } 547 \mathrm{~nm}$ between $2 \mu \mathrm{M} \mathrm{NO}_{2}$ and Griess reagent $(n=3)$. The standard deviations are represented as error bars.

\subsection{Reproducibly of the PEDD}

The reproducibility of the PEDD was determined by injecting a $2 \mu \mathrm{M}$ nitrite standard ten times. As shown in Figure 6 good reproducibility was achieved with a relative standard deviation of $2.5 \%$ calculated. An average change in response (peak height, $\mu \mathrm{s})$ of $63.1 \pm 1.6 \mu \mathrm{s}(n=3)$ was achieved.

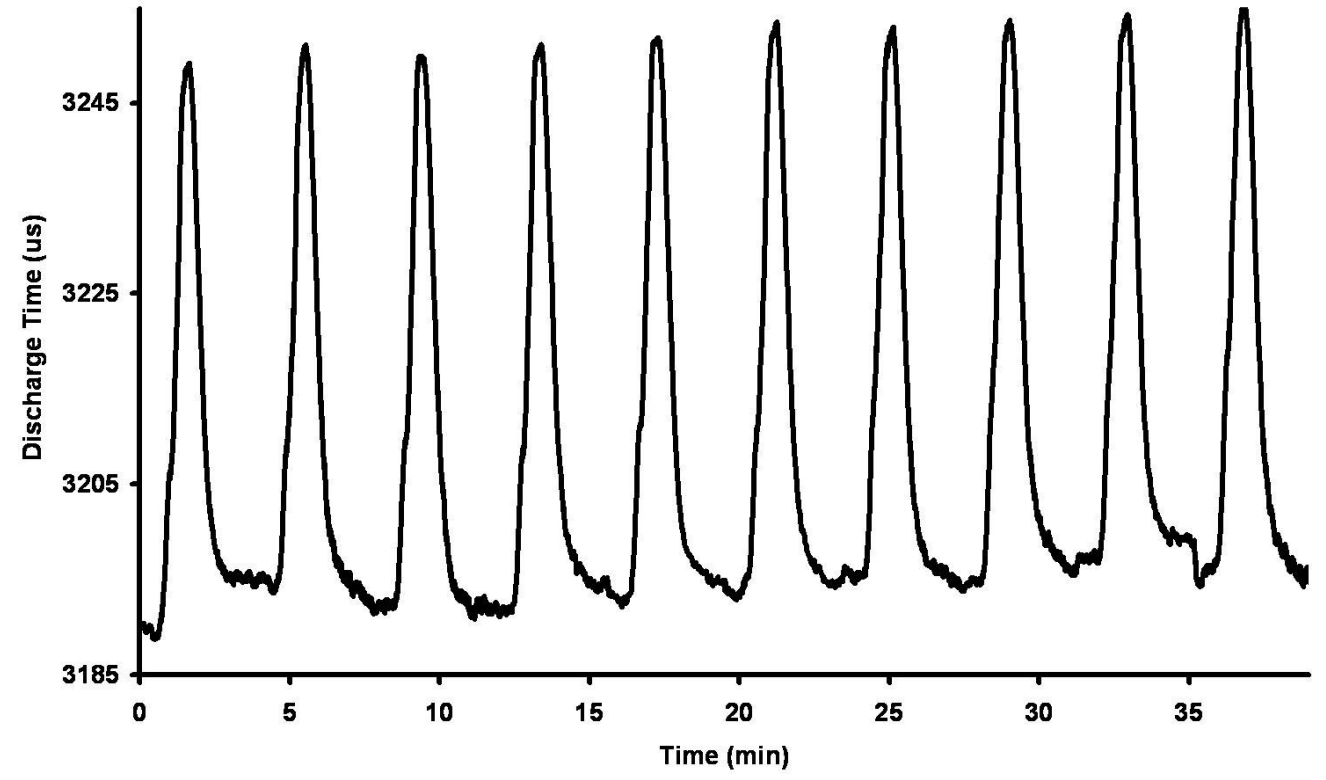

Figure 6 Determination of the reproducibility of the PEDD for the detection of $2 \mu \mathrm{MO}_{2} \mathrm{Griess}$ reagent complex $(n=10)$.

The reproducibility of PEDDs within a batch was investigated by fabricating 2 identical PEDDs (Emitter LED $\lambda_{\max } 530$ $\mathrm{nm}$ and detector LED $\left.\lambda_{\max } 623 \mathrm{~nm}\right)$. A $5 \mu \mathrm{M} \mathrm{NO}_{2}$ standard was injection into the system $(n=3)$ and monitoring using the first PEDD. The experiment was then repeated using the second PEDD $(n=3)$. An average peak height of $38.9 \pm$ 
$0.3 \mu$ s (R.S.D. $=0.7 \%$ ) for the detection of $5 \mu \mathrm{M}$ nitrite and Griess reagent complex was obtained from the first PEDD. An average peak height of $40.4 \pm 0.7 \mu$ s (R.S.D. $=1.8 \%$ ) for the detection of $5 \mu \mathrm{M}$ nitrite and Griess reagent complex was obtained from the second PEDD. This resulted in an average peak height of $39.7 \pm 1.1 \mu \mathrm{s}(\mathrm{R} . \mathrm{S}$.D. $=2.7 \%$ ) for the detection of $5 \mu \mathrm{M}$ nitrite and Griess reagent complex calculated using the two PEDDs. This demonstrated that the PEDDs can be fabricated reproducibably.

\subsection{Calibration of the PEDD flow cell and the $\mu$ Quant ${ }^{\mathrm{TM}}$ platewell reader}

Working nitrite calibration standards between 0.07 and $100 \mu \mathrm{M}$ were prepared from the $0.1 \mathrm{M}$ stock standard. Various concentrations of nitrite were prepared in deionised water and passed through the PEDD flow cell for ca. 5 minutes per sample, at a flow rate of $0.6 \mathrm{~mL} \mathrm{m^{-1 }}$. The $\log$ of the discharge times $(\log t, \mu \mathrm{s})$ was plotted against the nitrite Griess reagent complex concentration $(C)$ in accordance with the model (Equation 1) and the results are presented in Figure 7. A linear range of approximately $0.2-100 \mu \mathrm{M}$ nitrite Griess reagent complex $\left(R^{2}\right.$ value 0.9995$)$ was obtained as shown in Table 1. The relative standard deviation of the measurements $(n=3$, shown as error bars) is very low (ca. $0.01 \%)$. An LOD of ca. $7 \mathrm{nM}$ nitrite Griess reagent complex was achieved with a peak height of $4.7 \pm 1.8 \mu$ s (Baseline standard deviation, $3 \sigma=0.51 \mu \mathrm{s})$

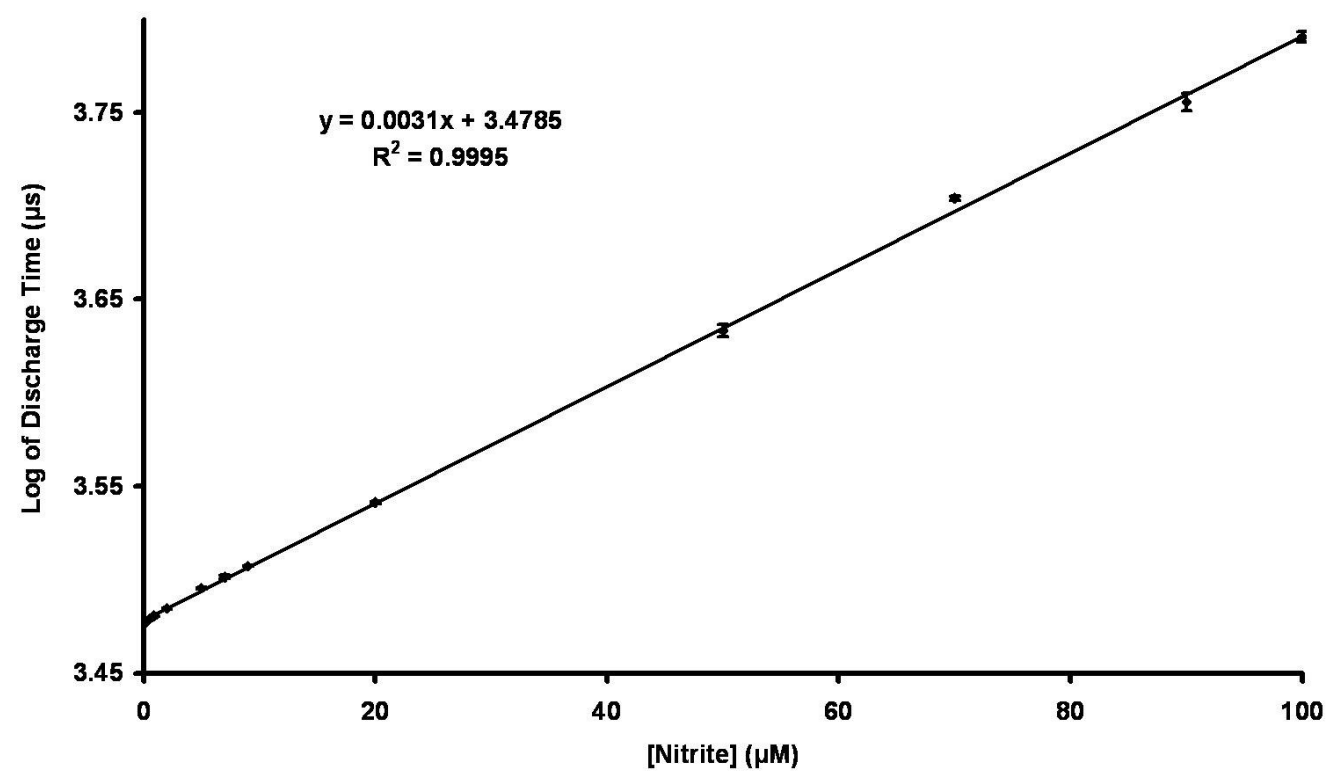

Figure 7 Log of the discharge times $(t)$ obtained using a PEDD versus nitrite Griess reagent complex concentration. The error bars represent the standard deviations for $n=3$.

As a comparison study, the absorbance of the same nitrite Griess reagent complex concentrations were acquired employing a $\mu$ Quant ${ }^{\mathrm{TM}}$ platewell reader. As shown in Figure 8 the mean normalized maximum absorbance $\left(R / R_{\max }\right)$ obtained at a $\lambda_{\max }$ of $547 \mathrm{~nm}$ was plotted against nitrite Griess reagent complex concentration $(C)$ resulting in a linear range $\left(R^{2}\right.$ value 0.9973$)$ of $0.9-100 \mu \mathrm{M}$ with a highest R.S.D. $(n=3)$ of $0.03 \%$. A significantly higher LOD of $0.9 \mu \mathrm{M}$ was determined using the $\mu$ Quant ${ }^{\mathrm{TM}}$ platewell reader (Table 1). 


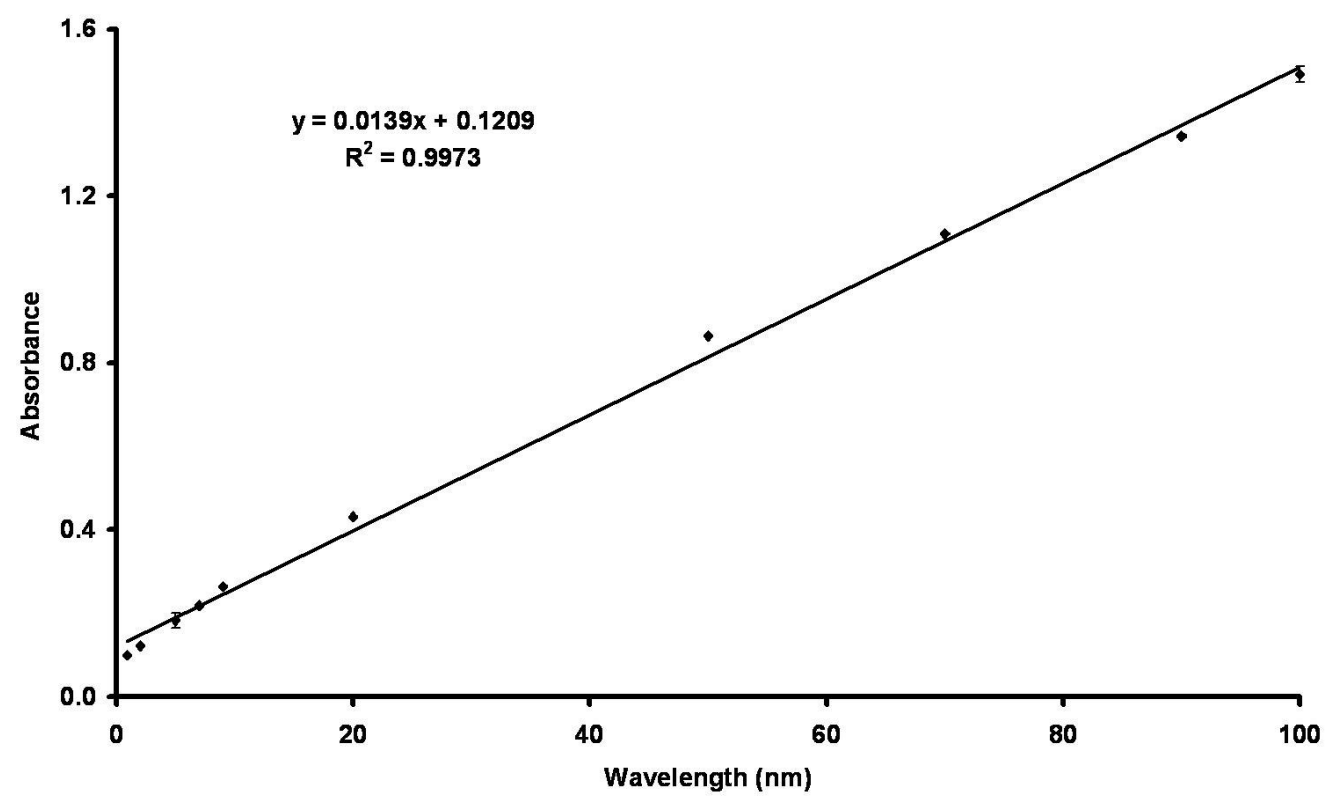

Figure 8 Absorbance obtained at a $\lambda_{\max }$ of $547 \mathrm{~nm}$ using a $\mu$ Quant ${ }^{\mathrm{TM}}$ platewell reader versus nitrite Griess reagent complex concentration. The error bars represent the standard deviations for $n=3$.

As outlined in Table 1 the PEDD achieved a lower limit of detection than a commercially available instrument. The PEDD also achieved improved precision $($ R.S.D. $=0.01 \%$ ) in comparison to the results calculated from the platewell reader $($ R.S.D. $=0.03 \%)$.

Table 1 A comparative summary of the data obtained for the detection of nitrite Griess reagent complex using both a PEDD and a $\mu$ Quant ${ }^{\mathrm{TM}}$ platewell reader $(n=3)$

\begin{tabular}{|l|c|c|}
\hline & PEDD $\left(\boldsymbol{\lambda}_{\max } \mathbf{5 3 0} \mathbf{~ n m}\right)$ & Platewell Reader $\left(\boldsymbol{\lambda}_{\max } \mathbf{5 4 7} \mathbf{~ n m}\right)$ \\
\hline Linear Range & $0.2-100 \mu \mathrm{M}$ & $0.9-100 \mu \mathrm{M}$ \\
$\mathbf{R}^{2}$ & 0.9995 & 0.9973 \\
LOD & $70 \mathrm{nM}$ & $0.9 \mu \mathrm{M}$ \\
\hline
\end{tabular}

\section{CONCLUSION}

The work presented here has demonstrated that the novel, low cost, miniaturized PEDD flow analysis system is highly sensitive for the detection of nitrite employing the Griess reaction method. The PEDD flow device achieved a linear range of $0.2-100 \mu \mathrm{M}$ and an LOD of $70 \mathrm{nM} \mathrm{NO}_{2}$. The commercially available bench top platewell reader achieved a linear range of $0.9-100 \mu \mathrm{M}$ and a higher LOD of $0.9 \mu \mathrm{M} \mathrm{NO}_{2}$. The PEDD was determined to be reproducible with a relative standard deviation of $2.5 \%$ for the detection of $2 \mu \mathrm{M}$ nitrite Griess reagent complex $(n=10)$. Data obtained from two PEDDs calculated a relative standard deviation of $2.7 \%$ for the detection of $5 \mu \mathrm{M}$ nitrite Griess reagent complex $(n=2)$. The PEDD flow device offers advantages of reproducibility, extremely low power consumption, no requirement for an A/D converter or operational amplifier and the sensor can be operated from a $9 \mathrm{~V}$ battery. This low cost detector can be applied to an autonomous remote sensing system for the monitoring of nitrite levels in situ.

\section{ACKNOWLEDGEMENTS}

The authors wish to thank Science Foundation Ireland SFI for grant support under the Adaptive Information Cluster Award (SFI 03/IN3/1361). We also acknowledge valuable help and support provided by Mitsubishi Electric Research Laboratories, Broadway, Cambridge, MA, USA. 


\section{REFERENCES}

[1] X.-F. Yue, Z.-Q. Zhang, H.-T. Yan, "Flow injection catalytic spectrophotometric simultaneous determination of nitrite and nitrate", Talanta 62 97-101, 2004

[2] M.J. Ahmed, C.D. Stalikas, S.M. Tzouwara-Karayanni, M.I. Karayannis, "Simultaneous spectrophotometric determination of nitrite and nitrate by flow-injection analysis", Talanta $\mathbf{4 3}$ 1009-1018, 1996

[3] A. Yamada, M. Sakuraba, J. Murota, "Integration of Si p-i-n diodes for light emitter and detector with optical waveguides", Materials Science in Semiconductor Processing 8 435-438, 2005

[4] T. Attin, K. Becker, C. Hannig, W. Buchalla, A. Wiegand, "Suitability of a malachite green procedure to detect minimal amounts of phosphate dissolved in acidic solutions", Clinical Oral Investigations 9 203-207, 2005

[5] Y. Miura, K. Kusakari, "Flow injection analysis of nitrite based on spectrophotometric measurements of iodine formed by oxidation of iodide with nitrite", Analytical Sciences 15 923-926, 1999

[6] R.S. Guerrero, C.G. Benito, J.M. Calatayud, "Flow-injection analysis-spectrophotometric determination of nitrite and nitrate in water samples by reaction with proflavin", Talanta $\mathbf{4 3}$ 239-246, 1996

[7] W. Yao, R.H. Byrne, R.D. Waterbury, "Determination of nanomolar concentrations of nitrite and nitrate in natural waters using long path length absorbance spectroscopy", Environmental Science Technology 32 2646-2649, 1998

[8] L. Monser, S. Sadok, G.M. Greenway, I. Shah, R.F. Uglow, "A simple simultaneous flow injection method based on phosphomolybdenum chemistry for nitrate and nitrite determinations in water and fish samples", Talanta 57 511-518, 2002

[9] S. Biswas, B. Chowdhury, B.C. Ray, "A novel spectrofluorimetric method for the ultra trace analysis of nitrite and nitrate in aqueous medium and its application to air, water, soil and forensic samples", Talanta 64 308-312, 2004

[10] R. Burakham, M. Oshima, K. Grudpan, S. Motomizu, "Simple flow-injection system for the simultaneous determination of nitrite and nitrate in water samples", Talanta 64 1259-1265, 2004

[11] E. Sawicki, T.W. Stanley, J. Pfaff, A. D'Amico, "Comparison of fifty-two spectrophotometric methods for the determination of nitrite", Talanta 10 641-655, 1963

[12] V.M. Ivanov, "The 125th Anniversary of the Griess Reagent", Journal of Analytical Chemistry $591002-$ 1005,2004

[13] M.J. Moorcroft, J. Davis, R.G. Compton, "Detection and determination of nitrate and nitrite: a review", Talanta 54 785-803, 2001

[14] M.B. Shinn, "Colorimetric method for determination of nitrite", Industrial and Engineering Chemistry: Analytical Edition 13 33-35, 1941

[15] P.C. Hauser, T.W.T. Rupasinghe, N.E. Cates, "A multi-wavelength photometer based on light-emitting diodes", Talanta 42 605-612, 1995

[16] M.A. Feres, B.F. Reis, "A downsized flow set up based on multicommutation for the sequential photometric determination of iron(II)/iron(III) and nitrite/nitrate in surface water", Talanta 68 422-428, 2005

[17] F.R.P. Rocha, B.F. Reis, "A flow system exploiting multicommutation for speciation of inorganic nitrogen in waters", Analytica Chimica Acta 409 227-235, 2000

[18] G.M. Greenway, S.J. Haswell, P.H. Petsul, "Characterisation of a micro-total analytical system for the determination of nitrite with spectrophotometric detection", Analytica Chimica Acta 387 1-10, 1999

[19] W. Frenzel, J. Schulz-Brussel, B. Zinvirt, "Characterisation of a gas-diffusion membrane-based optical flowthrough sensor exemplified by the determination of nitrite", Talanta, 2004

[20] E.T. Steimle, E.A. Kaltenbacher, R.H. Byrne, "In situ nitrite measurements using a compact spectrophotometric analysis system", Marine Chemistry 77 255-262, 2002

[21] L. Marle, G.M. Greenway, "Microfluidic devices for environmental monitoring", Trends in Analytical Chemistry 24 795-802, 2005

[22] O. Geschke, H. Klank, P. Tellemann, Microsystem Engineering of Lab-on-a-chip Devices, WILEY-VCH, 2004.

[23] A. Aydin, O. Ercan, S. Tascioglu, "A novel method for the spectrophotometric determination of nitrite in water", Talanta 66 1181-1186, 2005

[24] K.-T. Lau, S. Baldwin, M. O'Toole, R. Shepherd, W.J. Yerazunis, S. Izuo, S. Ueyama, D. Diamond, "A lowcost optical sensing device based on paired emitter-detector light emitting diodes", Analytica Chimica Acta 557 111-116, 2006

[25] M. O'Toole, K.-T. Lau, D. Diamond, "Photometric detection in flow analysis systems using integrated PEDDs", Talanta 66 1340-1344, 2005 
